Supplement of Biogeosciences, 13, 2859-2872, 2016

http://www.biogeosciences.net/13/2859/2016/

doi:10.5194/bg-13-2859-2016-supplement

(C) Author(s) 2016. CC Attribution 3.0 License.

(c) (i)

Supplement of

\title{
Equatorward phytoplankton migration during a cold spell within the Late Cretaceous super-greenhouse
}

Niels A. G. M. van Helmond et al.

Correspondence to: Niels A. G. M. van Helmond (n.vanhelmond@uu.nl)

The copyright of individual parts of the supplement might differ from the CC-BY 3.0 licence. 
Table S1. Geochemical and palynological data for Pratts Landing. In column four the number of counts of Cyclonephelium compactum-membraniphorum morphological plexus (Ccm) per sample is listed. In column five the percentage of the total dinocyst assemblage represented by $\mathrm{Ccm}$ is listed. In column six the absolute number of cysts of $\mathrm{Ccm}$ is listed in cysts per dry gram of sediment (cpg). N.D. $=$ not determined.

\begin{tabular}{|c|c|c|c|c|c|}
\hline Sample & $\begin{array}{l}\text { Height } \\
(\mathrm{m})\end{array}$ & $\begin{array}{c}\delta^{13} \mathrm{C} \\
(\% \text { VPDB })\end{array}$ & $\begin{array}{c}\mathrm{Ccm} \\
\text { (counts) }\end{array}$ & $\begin{array}{l}\mathrm{Ccm} \\
(\%)\end{array}$ & $\begin{array}{r}\mathrm{Ccm} \\
(\mathrm{cpg})\end{array}$ \\
\hline 1 & 0 & $-25,52$ & N.D. & N.D. & N.D. \\
\hline 2 & 0,5 & $-25,56$ & 1 & 0,5 & 41 \\
\hline 3 & 1 & $-25,35$ & N.D. & N.D. & N.D. \\
\hline 4 & 1.5 & -25.14 & N.D. & N.D. & N.D. \\
\hline 5 & 2 & -25.65 & 5 & 3.4 & 200 \\
\hline 6 & 2.5 & -25.32 & N.D. & N.D. & N.D. \\
\hline 7 & 3 & -25.34 & N.D. & N.D. & N.D. \\
\hline 8 & 3.5 & -25.39 & 6 & 4.0 & 174 \\
\hline 9 & 4 & -25.57 & N.D. & N.D. & N.D. \\
\hline 10 & 4.5 & -25.88 & N.D. & N.D. & N.D. \\
\hline 11 & 5 & -25.93 & 3 & 2.8 & 19 \\
\hline 12 & 5.5 & -25.86 & N.D. & N.D. & N.D. \\
\hline 13 & 6 & -25.25 & N.D. & N.D. & N.D. \\
\hline 14 & 6.5 & -25.14 & 7 & 3.6 & 433 \\
\hline 15 & 7 & -25.18 & 4 & 2.3 & 204 \\
\hline 16 & 7.5 & -25.40 & N.D. & N.D. & N.D. \\
\hline 17 & 8 & -25.33 & 1 & 0.6 & 83 \\
\hline 18 & 8.5 & -25.23 & N.D. & N.D. & N.D. \\
\hline 19 & 9 & -25.17 & 1 & 0.6 & 52 \\
\hline 20 & 9.2 & -25.23 & N.D. & N.D. & N.D. \\
\hline 21 & 9.4 & -24.85 & N.D. & N.D. & N.D. \\
\hline 22 & 9.6 & -25.16 & N.D. & N.D. & N.D. \\
\hline 23 & 9.8 & -25.01 & N.D. & N.D. & N.D. \\
\hline 24 & 10 & -25.13 & 4 & 1.8 & 277 \\
\hline 25 & 10.2 & -25.24 & N.D. & N.D. & N.D. \\
\hline 26 & 10.4 & -24.60 & N.D. & N.D. & N.D. \\
\hline 27 & 10.6 & -24.88 & N.D. & N.D. & N.D. \\
\hline 28 & 10.8 & -24.84 & N.D. & N.D. & N.D. \\
\hline 29 & 11 & -24.70 & 6 & 3.2 & 571 \\
\hline 30 & 11.2 & -24.64 & N.D. & N.D. & N.D. \\
\hline 31 & 11.4 & -24.83 & N.D. & N.D. & N.D. \\
\hline 32 & 11.6 & -24.50 & N.D. & N.D. & N.D. \\
\hline 33 & 11.8 & -24.35 & N.D. & N.D. & N.D. \\
\hline 34 & 12 & -23.89 & 32 & 18.7 & 5314 \\
\hline 35 & 12.2 & -24.30 & N.D. & N.D. & N.D. \\
\hline 36 & 12.4 & -24.34 & N.D. & N.D. & N.D. \\
\hline 37 & 12.6 & -23.62 & N.D. & N.D. & N.D. \\
\hline 38 & 12.8 & -24.08 & 3 & 1.8 & 699 \\
\hline 39 & 13 & -24.03 & N.D. & N.D. & N.D. \\
\hline 40 & 13.2 & -23.92 & N.D. & N.D. & N.D. \\
\hline 41 & 13.4 & -23.98 & 5 & 2.5 & 1059 \\
\hline 42 & 13.6 & -24.05 & N.D. & N.D. & N.D. \\
\hline 43 & 13.8 & -24.18 & N.D. & N.D. & N.D. \\
\hline 44 & 14 & N.D. & 26 & 12.9 & 5615 \\
\hline 45 & 14.2 & -23.81 & N.D. & N.D. & N.D. \\
\hline 46 & 14.4 & -24.14 & N.D. & N.D. & N.D. \\
\hline
\end{tabular}




\begin{tabular}{|c|c|c|c|c|c|}
\hline Sample & $\begin{array}{l}\text { Height } \\
\text { (m) }\end{array}$ & $\begin{array}{c}\delta^{13} \mathrm{C} \\
(\% \% \text { VPDB })\end{array}$ & $\begin{array}{c}\mathrm{Ccm} \\
\text { (counts) }\end{array}$ & $\begin{array}{l}C c m \\
(\%)\end{array}$ & $\begin{array}{l}C \mathrm{Cm} \\
(\mathrm{cpg})\end{array}$ \\
\hline 48 & 14.8 & -24.16 & N.D. & N.D. & N.D. \\
\hline 49 & 15 & -24.64 & 15 & 9.5 & 4913 \\
\hline 50 & 15.2 & -24.41 & N.D. & N.D. & N.D. \\
\hline 51 & 15.4 & -24.76 & N.D. & N.D. & N.D. \\
\hline 52 & 15.6 & -24.40 & N.D. & N.D. & N.D. \\
\hline 53 & 16 & -24.50 & 0 & 0.0 & 0 \\
\hline 54 & 16.2 & -24.44 & N.D. & N.D. & N.D. \\
\hline 55 & 16.4 & -24.74 & N.D. & N.D. & N.D. \\
\hline 56 & 16.6 & -24.77 & N.D. & N.D. & N.D. \\
\hline 57 & 16.8 & -24.08 & N.D. & N.D. & N.D. \\
\hline 58 & 17 & -24.77 & 2 & 1.7 & 438 \\
\hline 59 & 17.2 & -24.82 & N.D. & N.D. & N.D. \\
\hline 60 & 17.4 & -25.25 & N.D. & N.D. & N.D. \\
\hline 61 & 17.6 & -25.09 & N.D. & N.D. & N.D. \\
\hline 62 & 17.8 & -24.99 & N.D. & N.D. & N.D. \\
\hline 63 & & N.D. & 0 & 0.0 & 0 \\
\hline 64 & 18.2 & -25.51 & N.D. & N.D. & N.D. \\
\hline 65 & 18.4 & -25.72 & N.D. & N.D. & N.D. \\
\hline 66 & 18.6 & -25.91 & N.D. & N.D. & N.D. \\
\hline 67 & 18.8 & -25.61 & N.D. & N.D. & N.D. \\
\hline 68 & 19 & -25.04 & 6 & 5.5 & 3257 \\
\hline 69 & 19.2 & -25.66 & N.D. & N.D. & N.D. \\
\hline 70 & 19.4 & -25.58 & N.D. & N.D. & N.D. \\
\hline 71 & 19.6 & -25.72 & N.D. & N.D. & N.D. \\
\hline 72 & 19.8 & -25.57 & N.D. & N.D. & N.D. \\
\hline 73 & 20 & -25.75 & 8 & 5.7 & 404 \\
\hline 74 & 20.5 & -25.82 & N.D. & N.D. & N.D. \\
\hline 75 & 21.5 & -25.51 & N.D. & N.D. & N.D. \\
\hline 76 & 22 & -25.35 & 2 & 1.5 & 960 \\
\hline 77 & 22.5 & -25.95 & N.D. & N.D. & N.D. \\
\hline 78 & 23 & -25.98 & N.D. & N.D. & N.D. \\
\hline 79 & 23 & -25.93 & N.D. & N.D. & N.D. \\
\hline
\end{tabular}

